\title{
Prevención de la neumonía adquirida en la comunidad
}

\author{
MANUEL BARROS M.*, CLAUDIA CARTAGENA S.** y LUIS BAVESTRELLO F.***
}

\section{PRREVENTION OF COMMUNITY-ACQUIRED PNEUMONIA IN ADULTS}

Polysaccharide 23 valent pneumococcal vaccine commercially available from 1983 includes 23 serotypes of Streptococcus pneumoniae, representing near 90\% of strains involved in invasive pneumococcal disease in immune competent adults. Vaccine confers protection against invasive pneumococcal disease. Immunization is recommended in adults over 65 years old, in patients affected by chronic diseases (cardiopathies, COPD, nephropathies, diabetes mellitus, hepatic cirrhosis, chronic breakage in brain-blood barrier, functional or anatomical asplenia, alcoholism), in immunocompromised hosts, including HIV infection, chemotherapy treatment and hematological malignancies. Influenza vaccine is prepared with particulated antigens, including two influenza A strains and one influenza B strain, selected according to influenza epidemiological worldwide surveillance the year before. On account of continuous antigenic changes (drifts), it is necessary to modify the vaccine antigen's composition yearly. Cost/effectiveness evaluation has confirmed the efficacy of influenza vaccine in reducing morbidity and mortality associated to influenza epidemic and health economical resources involved in patient care. Besides, clinical trials have confirmed that immunization reduces the risk of acquiring pneumonia, of hospitalization and death in elderly people during the influenza epidemic, when vaccine antigenic composition is similar to the circulating strains. Vaccination is recommended annually in healthy adults over 65 years old, in patients with chronic diseases (cardiopathies, COPD, nephropathies, diabetes mellitus, hepatic cirrhosis, chronic breakage of blood-brain barrier, functional or anatomical asplenia, alcoholism). It is also recommended in women who will be in the second or third trimester of pregnancy during the influenza season, in immunocompromised hosts, in institutionalized patients (geriatrics), health care workers, and travelers to geographical areas that are affected by the influenza epidemic.

Key words: pneumonia, preventive measures, prophylaxis, prevention and control.

\section{RESUMEN}

La vacuna anti neumocóccica polivalente disponible desde 1983 incluye 23 serotipos de Streptococcus pneumoniae, que representan cerca de $90 \%$ de las cepas que ocasionan enfermedad neumocóccica invasora en adultos inmunocompetentes. La vacuna confiere protección contra la enfermedad neumocóccica invasora. Se recomienda vacunar a los adultos sanos sobre 65 años de edad, portadores de enfermedades crónicas (cardiopatías, EPOC, nefropatías, diabetes mellitus, cirrosis hepática, pérdida crónica de LCR, asplenia funcional o anatómica, alcoholismo), inmunocomprometidos incluyendo los infectados por VIH, quimioterapia y neoplasias

\footnotetext{
* Departamento de Medicina, Universidad de Valparaíso, Valparaíso, Chile.

** Hospital Carlos Van Buren, Valparaíso, Chile.

*** Servicio de Medicina, Hospital Gustavo Fricke, Viña del Mar, Chile.
} 
hematológicas. La vacuna anti influenza se prepara con antígenos particulados, e incluye dos tipos antigénicos de virus influenza A y una de influenza B, seleccionados de acuerdo al perfil epidemiológico que arroja la vigilancia mundial de influenza en el año anterior. Debido al cambio antigénico que se produce cada año, es necesario modificar la composición de la vacuna. Los estudios de costo-efectividad han confirmado la eficacia de la vacuna anti influenza en reducir la morbimortalidad asociada a la epidemia de influenza y los gastos de salud involucrados en el manejo de los enfermos. Además, los estudios clínicos han confirmado que la vacunación reduce el riesgo de neumonía, hospitalización y muerte en la población senescente durante la epidemia de influenza, cuando la cepa de la vacuna es similar a la presente en la comunidad. Se recomienda vacunar anualmente a los adultos sanos sobre 65 años de edad, portadores de enfermedades crónicas (cardiopatías, EPOC, nefropatías, diabetes mellitus, cirrosis hepática, pérdida crónica de LCR, asplenia funcional o anatómica, alcoholismo), mujeres embarazadas con más de 3 meses de gestación, inmunocomprometidos, pacientes institucionalizados (geriátricos, residentes en casas de reposo), trabajadores de la salud, viajeros a áreas geográficas de epidemia.

Palabras claves: neumonía, medidas preventivas, profilaxis, prevención y control.

\section{Medidas generales para prevenir la neumo- nía adquirida en la comunidad:}

- Acciones comunitarias: campañas de información en salud (evitar el hacinamiento, alcoholismo, tabaquismo, programas de vacunación).

- Acciones en el huésped: consejo nutricional, control de las enfermedades crónicas, vacunación de la población de riesgo.

\section{VACUNA ANTINEUMOCÓCICA}

La vacuna antineumocócica (VAN) polivalente disponible desde 1983 incluye 23 cepas de Streptococcus pneumoniae. En un estudio publicado en 1993, Butler y cols demostraron que la vacuna cubre cerca del $90 \%$ de las cepas que ocasionan enfermedad neumocócica invasora (ENI) en sujetos inmunocompetentes de 5 años o más ${ }^{1}$. La reciente introducción de la vacuna conjugada, contra 7 cepas que son prevalentes en la población infantil, fue evaluada por Whitney y $\operatorname{cols}^{2}$ analizando el registro de cepas de $S$. pneumoniae obtenidas de pacientes con ENI, a las que se determinó su sensibilidad. Se analizaron las tendencias en un área geográfica que incluye una población de 16 millones de habitantes, y estableció que la tasa de ENI disminuyó desde 24,3 por 100.000 habitantes (199899) a 17,3 por 100.000 (2001). Es interesante destacar que aunque la mayor caída ocurrió en la población menor de 2 años, en quienes está siendo aplicada la vacuna, también se registró una caída significativa en la población adulta, con un descenso de $32 \%$ en el grupo de 20-39 años, $8 \%$ en el grupo de 40-64 años, y $18 \%$ en los mayores de 65 años (tasa de 49,5 por 100.000 habitantes en el 2001 vs 60,1 por 100.000 en 1998-99). Otro aspecto de gran interés ha sido que la ENI ocasionada por cepas resistentes a penicilina, también disminuyó, desde 6,3 a 4,1 por 100.000 habitantes.

\section{Eficacia}

Moore y cols ${ }^{3}$ realizaron una revisión sistemática con el objetivo de establecer la eficacia de la vacuna antineumocócica, se examinaron 13 estudios clínicos aleatorios que enrolaron más de 45.000 personas [Ia]. En la revisión se comparó la población vacunada contra $S$. pneumoniae con aquella que no recibió la vacuna, estableciéndose que en personas inmunocompetentes (tres estudios clínicos aleatorios que reunieron 21.152 mineros de oro africanos y montañeses de Papúa, Nueva Guinea), la aplicación de la vacuna contra $S$. pneumoniae redujo significativamente la neumonía de cualquier etiología $(3,1$ vs 6,5\%; RR 0,56 IC95\%: 0,47-0,66), la neumonía neumocócica $(0,5$ vs $3,1 \%$; RR 0,16 IC95\%: 0,11-0,23), bacteriemia neumocócica (0,7 vs 3,8\%; RR 0,18 IC95\%: 0,09-0,34), y mortalidad relacionada con neumonía durante el invierno (1,1 vs 1,6\%; RR 0,70 IC95\%: 0,500,96). Sin embargo, en ancianos y en pacientes inmunocomprometidos (10 estudios clínicos aleatorios, 24.074 personas), no se encontró diferencias significativas entre ambos grupos.

En una revisión sistemática más reciente y más completa, realizada por el grupo Cochrane ${ }^{4}$, 
también encontró que la VAN tenía un efecto protector en la aparición de neumonía neumocócica, pero el resultado final estaba muy influido por un estudio publicado por Kaufmann y cols. en 1947 [Ia]. Este estudio y otros aparecidos algunos años después presentaban graves problemas metodológicos que dificultan la interpretación de sus resultados. De hecho, al excluir sólo este estudio del análisis, desaparecen los efectos beneficiosos de la vacunación en la población inmunocompetente. En resumen, al incluir en el análisis solamente los estudios más recientes, no se ha demostrado protección frente al desarrollo de neumonía con excepción del publicado por Ortqvist y cols ${ }^{5}$, lo que contrasta con el efecto protector que se había observado en los estudios más antiguos. Sin embargo, un problema de los estudios recientes es la menor incidencia de enfermedad confirmada, ya que sólo hubo 7 casos en los últimos 5 estudios, lo que disminuye el poder de los resultados.

Los datos disponibles no permiten distinguir si la heterogeneidad en los resultados se debe a cambios en la metodología de los ensayos e informes, diferencias en el lugar de los ensayos o a una pérdida de eficacia real de la vacuna en el curso del tiempo. Esto se debe a que los primeros ensayos, los cuales presentaban mayores problemas metodológicos, fueron realizados en población sana de alto riesgo, donde se espera que el beneficio fuera mayor ${ }^{4}$.

Una situación diferente ocurre al analizar la incidencia de ENI. Los únicos datos útiles entre los tres ensayos clínicos considerados en la revisión de Dear y cols ${ }^{4}$ son de Austrian (1976). Estos reflejan los resultados combinados de varios ensayos con denominadores totales de 3.953 sujetos vacunados y 8.024 controles, y un estudio de 13 valencias que incluyó solamente 1.493 sujetos vacunados y 3.002 controles. La vacuna tuvo un efecto protector significativo para la ENI (OR 0,18, IC95\%: 0,09-0,34, p < 0,001) [II].

El análisis de la mortalidad tiene un problema similar. La presencia de gran heterogeneidad en los estudios disponibles lleva a un intervalo de confianza muy amplio, por lo que la vacunación no se demuestra como protectora del riesgo medido.

La eficacia de la VAN en adultos mayores de 65 años fue recientemente analizada en un estudio de cohorte $^{6}$, donde 47.365 sujetos fueron seguidos [II]. En el período examinado, 26.313 sujetos recibieron inmunización y 21.052 no la recibieron. En el seguimiento de tres años, 1.428 pacientes fueron hospitalizados, 3.061 fueron tratados ambulatoriamente, y 61 presentaron bacte- remia. La vacunación se asoció a un menor riesgo de bacteriemia por neumonía (RR 0,56; IC95\%: 0,33-0,93) y un aumento del número de hospitalizaciones (RR 1,14; IC95\%: 1,02-1,28). No se modificó el riesgo de presentar neumonía en general (RR 1,07; IC95\%: 0,99-1,14) o neumonía tratada ambulatoriamente (RR 1,04; IC95\%: 0,96-1,13). En el grupo vacunado fue más frecuente el antecedente de tabaquismo, diabetes mellitus, cardiopatía coronaria, EPOC y visitas médicas ambulatorias en el año previo. Esto refleja la tendencia a proteger con la vacuna a la población que está más comprometida en su salud.

Los resultados obtenidos en el meta-análisis de estudios no aleatorios ${ }^{4}$, de los que se puede extraer información confiable, demostraron que la VAN otorga protección frente al desarrollo de ENI (OR 0,47; IC95\%: 0,37-0,59) [Ia]. En el análisis de subgrupos, se confirmó la protección en sujetos inmunocompetentes (OR 0,44; IC95\%: 0,34-0,58) y en adultos mayores inmunocompetentes (OR 0,30; IC95\%: 0,14-0,63).

La eficacia de la vacunación, en la prevención de la ENI, oscila entre 53 y 70\%. Cuando sólo se consideran los cuadros confirmados, con serotipos presentes en la vacuna, la eficacia aumenta a 56-75\%. El resumen de los estudios de casos y controles analizados demuestra una eficacia de la vacuna de un $53 \%$, lo que corresponde a un número necesario a tratar (NNT) de 20.000 individuos vacunados por infección grave que se evita y quizás 50.000 sujetos vacunados por muerte que se evita 4 .

Se recomienda administrar la vacuna antineumocócica (Tabla 1) a adultos mayores de 65 años, pacientes de cualquier edad portadores de enfermedades crónicas (enfermedades cardiovasculares, respiratorias y renales crónicas, diabetes mellitus, alcoholismo, daño hepático crónico, asplenia), e inmunocomprometidos [B].

Tabla 1. Indicaciones de la vacuna antineumocócica

Adultos sanos $\geq 65$ años

Portadores de enfermedades crónicas: cardiopatías, EPOC, nefropatías, diabetes mellitus, cirrosis hepática, pérdida crónica de LCR, asplenia funcional o anatómica, alcoholismo, tabaquismo

Inmunocomprometidos, incluyendo infección por VIH. 
Tabla 2. Recomendaciones de revacunación antineumocócica según categorías de riesgo

\begin{tabular}{|c|c|}
\hline Categorías de riesgo & Recomendación de revacunación \\
\hline Adulto mayor de 65 años & $\begin{array}{l}\text { Sólo aquellos que recibieron la } 1^{\mathrm{a}} \text { dosis antes de los } 65 \\
\text { años y se vacunaron hace más de } 5 \text { años. }\end{array}$ \\
\hline $\begin{array}{l}\text { Portadores de enfermedades crónicas (cardiopatías, } \\
\text { EPOC, nefropatías, hepatopatías, etc.) }\end{array}$ & No se recomienda administrar una $2^{\mathrm{a}}$ dosis. \\
\hline $\begin{array}{l}\text { Pacientes inmunocomprometidos } \\
\text { (mayores de } 2 \text { años de edad) }\end{array}$ & $\begin{array}{l}\text { Revacunar a los } 5 \text { años. Administrar la } 2^{\mathrm{a}} \text { dosis luego de } \\
3 \text { años si el paciente tenía menos de } 10 \text { años al recibir la } \\
\text { primera dosis. }\end{array}$ \\
\hline
\end{tabular}

\section{Revacunación}

El fundamento de la revacunación se basa en la demostración de una caída progresiva del nivel de anticuerpos después de 3 a 5 años de haber recibido la vacuna, y que en pacientes de alto riesgo y población senescente se ha observado un descenso de los títulos de anticuerpos entre los 6 y 9 años $^{7}$ [III]. Por otra parte, la revacunación sólo recupera los niveles de anticuerpo a títulos cercanos al $40 \%$ del nivel original $^{8}$.

Por ello, en Estados Unidos de América ${ }^{9}$ se recomienda revacunar solamente a los mayores de 65 años, siempre que hayan recibido la dosis previa antes de los 65 años (y no menos de 5 años atrás), mientras que en el Reino Unido solamente se recomienda suministrarla a aquellos individuos en riesgo de disminuir sus títulos de anticuerpos luego de 5 a 10 años $^{10}$ [C]. No debe administrarse dentro de los primeros 3 años por la posibilidad de reacciones adversas serias, asociado a un elevado título de anticuerpos circulantes (Tabla 2) ${ }^{10}$.

En el adulto mayor, el efecto protector conferido por la VAN no se modifica según el mo-

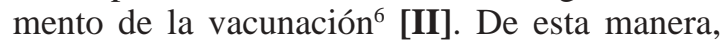
el efecto fue similar en aquellos individuos vacunados durante los últimos 12 meses (OR 1,08; IC95\%: 0,87-1,33) o si habían transcurrido 36 meses o más (OR 1,18; IC95\%: 1,04-1,34).

\section{Reacciones adversas}

La vacuna antineumocócica contiene $25 \mathrm{mg}$ de polisacárido capsular purificado de 23 serotipos capsulares de $S$. pneumoniae. Se administra una dosis de $0,5 \mathrm{ml}$ vía subcutánea o preferentemente intramuscular en el músculo deltoides o la porción lateral del muslo. La vacuna es segura [II]. En una de las revisiones sistemáticas mencionadas previamente ${ }^{3}$ se encontraron escasas referencias sobre los efectos ad- versos. Ocasionalmente, la vacunación contra el neumococo se ha asociado a reacciones cutáneas, tales como eritema, tumefacción, dolor e inflamación local y fiebre.

\section{VACUNA ANTIINFLUENZA}

\section{Eficacia}

La vacuna antiinfluenza se prepara con antígenos particulados, incluyendo habitualmente dos cepas de virus influenza $A$ y una de influenza B, seleccionadas de acuerdo al perfil epidemiológico del año respectivo. Debido al cambio antigénico que se produce cada año, es necesario modificar la composición de la vacuna. La eficacia depende de múltiples factores, entre otros, la coincidencia del virus presente en la comunidad con el incluido en la vacuna, factores ambientales, y factores del huésped. Se estima que en un adulto sano, la eficacia varía entre 70 y $90 \%$, en sujetos mayores de 60 años, no institucionalizados, es de $58 \%$ y, en adultos mayores residentes en centros geriátricos disminuye a 30-40\% [II]. En un meta-análisis que examinó veinte estudios de cohorte, publicado por Gross y cols. en $1995^{11}$, se demostró que la eficacia variaba de acuerdo a los resultados que querían obtenerse: la prevención de la enfermedad respiratoria fue de 56\% (IC95\%: 39 a 68\%); la neumonía fue de 53\% (IC95\%: 35 a 66\%); la admisión al hospital fue de 50\% (IC95\%: 28 a $65 \%$ ) y, el riesgo de muerte fue de $68 \%$ (IC95\%: 56 a $76 \%$ ) [Ia]. La eficacia de la vacuna en los estudios de casos y controles varió entre 32 y $45 \%$ para prevenir la hospitalización por neumonía, 31 a $65 \%$ para prevenir muertes hospitalarias por neumonía e influenza, 43 a $50 \%$ para prevenir muerte en el hospital por cualquier enfermedad respiratoria y 27 a $30 \%$ para prevenir muerte por cualquier causa [II, III]. Los 
estudios de costo-efectividad han confirmado la eficacia de la vacuna antiinfluenza en reducir la morbimortalidad asociada a la epidemia de influenza y la disminución de los gastos de salud involucrados en el manejo de los enfermos. Además, los estudios clínicos han confirmado que la vacunación reduce el riesgo de neumonía, la hospitalización y muerte de la población senescente durante la epidemia de influenza, cuando la cepa de la vacuna es similar a la presente en la comunidad.

Se recomienda vacunar anualmente contra la influenza a los adultos mayores ( $\geq 65$ años), portadores de enfermedades crónicas de cualquier edad (enfermedades cardiovasculares, respiratorias, renal y hepática crónicas, diabetes mellitus) y pacientes inmunocomprometidos (Tabla 3) [A]. En la campaña anual de vacunación deben participar todos los médicos, el equipo de salud y organizaciones de salud pública.

Ahmed y cols ${ }^{12}$ demostraron la eficacia de la vacuna en sujetos mayores de 16 años, observando una reducción promedio de la mortalidad de un 41\%; los autores destacan que la reducción fue sólo de $9 \%$ si los sujetos recibían la vacuna por primera vez, y $75 \%$ si habían sido vacunados antes, lo que sugiere un mayor beneficio si se recibe la vacuna en años sucesivos [III]. En un estudio observacional, Fedson y cols $^{13}$ demostraron en sujetos mayores de 45 años vacunados una reducción de $30 \%$ en la admisión al hospital, y 43-65\% en el riesgo de muerte en el hospital [III]. Por otra parte, en ancianos se reduce a la mitad la incidencia de influenza clínica y de complicaciones en la evolución de la enfermedad ${ }^{14}$ [II]. En pacientes con patología pulmonar crónica la vacunación disminuye también el número de consultas ambulatorias ${ }^{15}$ [III] .

Nichol y cols ${ }^{16}$ estudiaron recientemente una cohorte de sujetos adultos mayores de 65 años, durante dos períodos de influenza (1998-1999 y 1999-2000), revisando el impacto de la vacunación en el riesgo de hospitalización por cardiopatía, accidente vascular cerebral, neumonía o influenza, y muerte por cualquier causa [II]. Las cohortes fueron integradas por 140.055 sujetos (1998-1999) y 146.328 (1999-2000) y la cobertura de la vacunación obtenida fue de 55,5 y $59,7 \%$ respectivamente. Los sujetos vacunados tenían más enfermedades concurrentes, consultas médicas y hospitalizaciones por neumonía comparado con aquellos no vacunados, reflejando que el énfasis en la campaña de vacunación estuvo precisamente en la población de mayor riesgo. Sin embargo, en el grupo no va- cunado había más sujetos con diagnóstico de demencia o accidente vascular cerebral. La vacunación se asoció con una disminución del riesgo de hospitalización por cardiopatía (19\% en ambas temporadas), enfermedad cerebrovascular (16 y $23 \%$, respectivamente), y por neumonía o influenza (32 y $29 \%$, respectivamente). Se demostró además una disminución del riesgo de muerte, por cualquier causa de un 48 y $50 \%$. Estos hallazgos fueron similares en los distintos grupos etáreos, en presencia o ausencia de enfermedades concomitantes significativas y en los diferentes sitios en que se recogieron los datos.

Si se estima el número de tratamientos requeridos para prevenir un episodio (NNT), se observa que para prevenir una hospitalización por neumonía o influenza el NNT varía entre 347 y 431 (primera y segunda cohorte), para prevenir una muerte entre 95 y 118 , y para prevenir cualquier efecto clínico pernicioso entre $61 \mathrm{y}$ 68 , confirmando la enorme importancia de la vacunación antiinfluenza en los mayores de 65 años.

Armstrong y cols estudiaron el impacto de la vacunación antiinfluenza en el riesgo de muerte de los adultos mayores de 75 años ${ }^{17}$ [II]. Para ello estudiaron una cohorte prospectiva de 24.535 personas en 75 centros de salud del Reino Unido, a quienes invitaron a participar en un seguimiento de vacunación selectiva versus vacunación universal, y utilizando los datos de vigilancia epidemiológica establecieron la mortalidad en las temporadas de alta y baja circulación del virus influenza. Los resultados demostraron una menor mortalidad en el grupo vacunado, que no se incrementaba durante la época de alta circulación de virus, a diferencia de lo ocurrido en los no vacunados, en que la mortalidad casi se duplicó. La proporción de muertes atribuibles a influenza fue $13,4 \%$ en sujetos no vacunados, y sólo $2,2 \%$ en aquellos que recibieron la vacuna. Sobre la base de esas cifras, la eficacia de la vacuna para prevenir muerte por cualquier causa fue estimada en 83\% (IC 95\%: 9 a 100\%), pero el rango de confianza es demasiado amplio para permitir realizar conclusiones definitivas.

Los estudios de costo eficacia han sido escasos, y no necesariamente son extrapolables a otros países, especialmente por las diferencias en el costo de las hospitalizaciones entre Estados Unidos de América y otras naciones. Mullooly y cols ${ }^{18}$ estudiaron una cohorte de adultos mayores, que dividieron en población de "alto riesgo" y "no alto riesgo", de acuerdo con la presencia de comorbilidad [II]. Ellos obtuvieron una cobertura de 32 y $22 \%$, respectivamente. 
La eficacia en la prevención de hospitalización por neumonía o influenza fue de $30 \%$ (IC95\%: 17 a $42 \%$ ) para el grupo de alto riesgo y $40 \%$ (IC95\%: 1 a 64\%) en la otra categoría. El ahorro neto estimado por individuo vacunado fue de US $\$ 6,11$ para el primer grupo y de US $\$ 1,10$ si se consideran todos los ancianos. El costo neto por cada individuo vacunado en el grupo que no presentaba alto riesgo fue de US $\$ 4,82$.

Nichol y cols ${ }^{19}$ examinaron tres cohortes de adultos mayores con más de 20.000 sujetos cada una, que recibieron la vacuna antigripal en las temporadas 1990-91, 1991-92 y 1992-93 [II]. La cobertura de la vacunación varió entre 45 y $58 \%$, y aunque los sujetos vacunados tenían más enfermedades concomitantes, presentaron una disminución significativa de la tasa de hospitalizaciones por neumonía e influenza (entre 48 y $57 \%, \mathrm{p}<0,005)$ y por patología respiratoria (entre 27 y $39 \%, \mathrm{p}<0,01$ ). La vacunación se asoció también con una disminución de $37 \%$ en las hospitalizaciones por insuficiencia cardíaca en la epidemia de 1991-1992. Los costos de hospitalización fueron menores en el grupo vacunado en el período de 1991-92 (rango de 47 a $66 \%, \mathrm{p}<0,005)$ y para las hospitalizaciones por patología respiratoria y cardíaca en el período 1990-91 (disminución de 37 y 43\%, respectivamente; $\mathrm{p}<0,05)$. La vacunación se asoció también con reducción de la mortalidad general de 39 a 54\% en las tres temporadas de influenza ( $p<0,001)$. Los ahorros netos estimados en esta investigación fueron de US \$117 por persona vacunada (rango de US \$21 a US \$235).

\section{Reacciones adversas}

Además de las molestias locales, que son leves y transitorias, se ha investigado si la administración de la vacuna puede provocar efectos respiratorios adversos. Diversos estudios han demostrado que no hay riesgo de efectos respiratorios adversos en ancianos sanos ${ }^{20,21}$, ni tampoco riesgo de exacerbación en asmáticos menores de 65 años $^{22}$ ni en mayores de 65 años con asma o $\mathrm{EPOC}^{23}[\mathbf{I} \mathbf{b}, \mathbf{I I}]$.

El riesgo de exacerbación de enfermedades respiratorias crónicas fue estudiado en asmáticos menores de 65 años por un grupo de investigadores reunidos en los Centros de Investigación Clínica de Asma $^{22}$ [Ib]. Ellos compararon un registro diario de síntomas en los 15 días posteriores a la administración de placebo o vacuna, en un estudio randomizado, doble ciego y cruzado, que incluyó 2.032 pacientes, entre 3 y 64 años. Las exacerbaciones de asma fueron similares en ambos períodos (28,8 y 27,7 en los
Tabla 3. Indicaciones de la vacuna antiinfluenza

Adultos sanos $\geq 65$ años

Portadores de enfermedades crónicas: cardiopatías, EPOC, nefropatías, diabetes mellitus, cirrosis hepática, pérdida crónica de LCR, asplenia funcional o anatómica, alcoholismo, tabaquismo.

Embarazada con más de 3 meses de gestación

Inmunocomprometidos

Pacientes institucionalizados (geriátricos, casas de reposo, etc.)

Trabajadores de la salud

Cuidadores de sujetos de riesgo elevado

Viajeros a áreas geográficas de epidemia

sujetos que recibieron o no la vacuna). Más recientemente se publicó un artículo que abordó el mismo problema en los pacientes mayores de 65 años $^{23}$, tanto asmáticos como portadores de EPOC, ya que existía evidencia limitada en la literatura, con estudios no concluyentes por su poder insuficiente ${ }^{24,25}$. Los autores estudiaron pacientes incluidos en la base de datos de los médicos generales del Reino Unido, extrayendo de las fichas de los pacientes con diagnóstico de asma o EPOC, el antecedente de exacerbación de ambas enfermedades y las prescripciones de corticoesteroides orales [II]. La cobertura de la vacunación fue de $41 \%$ (39-43\%) en asmáticos y $33 \%(31-35 \%)$ en portadores de EPOC. Los resultados demostraron que no hubo diferencias entre el grupo vacunado (4.652) y no vacunado (7.348) en relación con la tasa de exacerbaciones y prescripciones de corticoesteroides orales al comparar los seis meses previos a la vacunación con los seis meses posteriores a ésta.

\section{VACUNACIÓN MIXTA ANTINEUMOCÓCICA Y ANTIINFLUENZA}

Diferentes estudios han abordado la eficacia de la vacunación simultánea para $S$. pneumoniae e influenza, considerando que los grupos de riesgo son similares. En un estudio de cohorte prospectivo no concurrente, Nichol y cols ${ }^{26}$ demostraron una reducción significativa en la tasa de hospitalización por influenza o neumonía, y la mortalidad atribuible en sujetos que recibieron la vacunación mixta, comparados con aquellos que solamente recibieron la VAN [II]. La VAN 
no confirió una mayor protección para el primer objetivo, pero redujo un $29 \%$ el riesgo de muerte (IC 95\%: 9-44\%), mientras que el grupo con vacunación mixta tuvo una protección de $72 \%$ (IC95\%: 42-86\%) y 82\% (IC95\%: 42-89\%), respectivamente.

En un estudio de intervención a gran escala, un grupo de residentes en Estocolmo, mayores de 65 años, fue invitado por correo a participar en una campaña de vacunación contra la influenza y $S$. pneumoniae ${ }^{27}$ [II]. El diseño del estudio fue prospectivo, con tres años de seguimiento, de los 259.627 sujetos invitados, 100.242 (39\%) fueron vacunados. Los resultados analizados fueron muerte por influenza, por neumonía, por neumonía neumocócica y por ENI. La tasa de incidencia (por 100.000 habitantes por año) de admisión al hospital fue menor en el grupo vacunado: 263 versus 484 (-46\%; IC95\%: -34 a $-56 \%)$ para influenza; 2.199 versus 3.097 (-29\%; IC95\%: -24 a -34\%) para neumonía; 64 versus 100 (-36\%; IC95\%: -3 a -58\%) para neumonía neumocócica, y 20 versus 40 (-52\%; IC95\%: ${ }^{-1}$ a $-77 \%$ ) para ENI. La mortalidad general disminuyó a la mitad en el grupo vacunado, $(15,1 \mathrm{vs}$ 34,7 muertes por 1.000 habitantes).

Por no haberse planeado este estudio con randomización es posible que el estado de salud basal haya sido diferente, pero en general, estudios previos han demostrado que el grupo vacunado corresponde a la población más enferma, por lo que no se invalidan los resultados. Es posible también que el grupo no vacunado haya recibido la VAN en años previos, o incluso en el mismo año -fuera del programa- y contar por lo tanto, con protección. De la misma manera anterior, esto implicaría un sesgo a favor del grupo no vacunado, y por lo tanto no invalida los resultados obtenidos.

Un estudio más reciente, del mismo grupo, comparó los resultados de la vacunación antiinfluenza y la VAN en sujetos mayores de 65 años $^{28}$ [II]. En 1999 a todos los adultos mayores de Estocolmo $(\mathrm{n}=258.754)$ se les ofreció participar en el programa de vacunación antiinfluenza y antineumocócica. El objetivo de esta investigación prospectiva fue determinar la eficacia de las vacunas en disminuir la necesidad de tratamiento hospitalario y muerte por influenza, neumonía y ENI. Para ello se revisaron los respectivos registros durante el año siguiente a la campaña. Fueron vacunados 124.702 sujetos (48\%), 72.107 con ambas vacunas, 29.346 solamente recibieron la vacuna antiinfluenza y 23.249 la vacuna antineumocócica. Se observó una disminución significativa de la tasa de hospitalización en el grupo vacunado. Los autores destacan la presencia de un efecto aditivo en la eficacia, cuando se administraron ambas vacunas, con reducción de los ingresos hospitalarios por influenza (37\%), neumonía (29\%) y ENI (44\%). También la mortalidad hospitalaria fue menor en los que recibieron las dos vacunas, comparados con aquellos que fueron vacunados con solamente una de ellas.

Otro estudio que abordó la eficacia de la vacunación combinada ${ }^{6}$, reveló que la vacunación contra la influenza se asoció con una disminución significativa del riesgo de hospitalización por neumonía adquirida en la comunidad (RR 0,78; IC 95\%: 0,65 a 0,95) y el riesgo de muerte (RR 0,68; IC95\%: 0,62 a 0,76) en la temporada de influenza [III]. Sin embargo, no tuvo relación con la incidencia de neumonía de manejo ambulatorio (RR 1,12; IC 95\%: 0,97 a 1,30) o bacteriemia neumocócica (RR 0,92; IC95\%: 0,38 a 2,25). En adultos inmunocompetentes, con o sin enfermedad pulmonar crónica, no hubo beneficio al añadir la VAN a aquellos que reciben anualmente la vacuna contra la influenza.

Aún no ha sido esclarecido, si la administración simultánea de ambas vacunas en adultos mayores reduce la tasa de hospitalización, la ENI y el riesgo de muerte. Aparentemente, el grado de protección conferido por la vacuna mixta aumenta con la edad [B].

\section{BIBLIOGRAFIA}

1.- BUTLER J C, BREIMAN R F, CAMPBELL J F, LIPMAN H B, BROOME C V, FACKLAM R R. Pneumococcal polysaccharide vaccine efficacy. An evaluation of current recommendations. JAMA 1993; 270: 1826-31.

2.- WHITNEY C G, FARLEY M M, HADLER J, HARRISON L H, BENNETT N M, LYNFIELD R, et al. Active Bacterial Core Surveillance of the Emerging Infections Program Network. Decline in invasive pneumococcal disease after the introduction of protein-polysaccharide conjugate vaccine. $\mathrm{N}$ Engl J Med 2003; 348: 1737-46.

3.- MOORE R A, WIFFEN P J, LIPSKY B A. Are the pneumococcal polysaccharide vaccines effective? Meta-analysis of the prospective trials. BMC Family Practice 2000; 1: 1. http://www.biomedcentral.com/ 1471-2296/1/1

4.- DEAR K, HOLDEN J, ANDREWS R, TATHAM D. Vaccines for preventing pneumococcal infection in adults. Cochrane Database Syst Rev 2003; 4: CD000422.

5.- ORTQVIST A, HEDLUND J, BURMAN L A, ELBEL E, HOFER M, LEINONEN M, et al. Randomised trial of 23-valent pneumococcal capsular polysaccharide vaccine in prevention of pneumonia in middle-aged and elderly people. Swedish Pneumococcal Vaccination Study Group. Lancet 1998; 351: 399-403. 
6.- JACKSON L A, NEUZIL K M, YU O, BENSON P, BARLOW W E, ADAMS A L, et al. Vaccine Safety Datalink. Effectiveness of pneumococcal polysaccharide vaccine in older adults. N Engl J Med 2003; 348: 1747-55.

7.- HEDLUND J U, KALIN M E, ORTQVIST A B, HENRICHSEN J. Antibody response to pneumococcal vaccine in middle-aged and elderly patients recently treated for pneumonia. Arch Intern Med 1994; 154: 1961-5.

8.- MUSHER D M, GROOVER J E, ROWLAND J M, WATSON D A, STRUEWING J B, BAUGHN R E, et al. Antibody to capsular polysaccharides of Streptococcus pneumoniae: prevalence, persistence, and response to revaccination. Clin Infect Dis 1993; 17: 66-73.

9.- ANÓNIMO. Prevention and control of influenza: recommendations of the Advisory Committee on Immunization Practices (ACIP). Centers for Disease Control and Prevention. MMWR Recomm Rep 1998; 47 (RR-6): 1-26.

10.- BRITISH THORACIC SOCIETY STANDARDS OF CARE COMMITTEE. BTS Guidelines for the Management of Community Acquired Pneumonia in Adults. Thorax 2001; 56 (Suppl 4): iv1-iv64.

11.- GROSS P A, HERMOGENES A W, SACKS H S, LAU J, LEVANDOWSKI R A. The efficacy of influenza vaccine in elderly persons. A meta-analysis and review of the literature. Ann Intern Med 1995; 123: 518-27.

12.- AHMED A E, NICHOLSON K G, NGUYEN-VANTAM J S. Reduction in mortality associated with influenza vaccine during 1989-90 epidemic. Lancet 1995; 346: $591-5$

13.- FEDSON D S, WAJDA A, NICOL J P, HAMMOND G W, KAISER D L, ROOS D L. Clinical effectiveness of influenza vaccination in Manitoba. JAMA 1993; 270: 1956-61.

14.- GOVAERT $\mathrm{T} M$, THIJS $\mathrm{C} T$, MASUREL $\mathrm{N}$, SPRENGER M J, DINANT G J, KNOTTNERUS J A. The efficacy of influenza vaccination in elderly individuals. A randomized double-blind placebocontrolled trial. JAMA 1994; 272: 1661-5.

15.- NICHOL KL, BAKEN L, NELSON A. Relation between influenza vaccination and outpatient visits, hospitalization, and mortality in elderly persons with chronic lung disease. Ann Intern Med 1999; 130: 397 403.

16.- NICHOL K L, NORDIN J, MULLOOLY J, LASK R, FILLBRANDT K, IWANE M. Influenza vaccination and reduction in hospitalizations for cardiac disease and stroke among the elderly. N Engl J Med 2003; 348: $1322-32$.

17.- ARMSTRONG B G, MANGTANI P, FLETCHER A, KOVATS S, McMICHAEL A, PATTENDEN S, et al.
Effect of influenza vaccination on excess deaths occurring during periods of high circulation of influenza: cohort study in elderly people. BMJ 2004; 329: 660.

18.- MULLOOLY J P, BENNETT M D, HORNBROOK M C, BARKER W H, WILLIAMS W W, PATRIARCA P A, et al. Influenza vaccination programs for elderly persons: cost-effectiveness in a health maintenance organization. Ann Intern Med 1994; 121: 947-52.

19.- NICHOL K L, MARGOLIS K L, WUORENMA J, VON STERNBERG T. The efficacy and cost effectiveness of vaccination against influenza among elderly persons living in the community. N Engl J Med 1994; 331: 778-84.

20.- GOVAERT T M, DINANT G J, ARETZ K, MASUREL N, SPRENGER M J, KNOTTNERUS J A. Adverse reactions to influenza vaccine in elderly people: randomised double blind placebo controlled trial. BMJ 1993; 307: 988-90.

21.- MARGOLIS K L, NICHOL K L, POLAND G A, PLUHAR R E. Frequency of adverse reactions to influenza vaccine in the elderly. A randomized, placebocontrolled trial. JAMA 1990; 264: 1139-41.

22.- ANÓNIMO. The safety of inactivated influenza vaccine in adults and children with asthma. From the American Lung Association Asthma Clinical Research Centers. N Engl J Med 2001; 345: 1529-36.

23.- TATA L J, WEST J, HARRISON T, FARRINGTON P, SMITH C, HUBBARD R. Does influenza vaccination increase consultations, corticosteroid prescriptions, or exacerbations in subjects with asthma or chronic obstructive pulmonary disease?. Thorax 2003; 58: 8359.

24.- CATES C J, JEFFERSON T O, BARA A I, ROWE B H. Vaccines for preventing influenza in people with asthma (Cochrane Review). Cochrane Database Syst Rev 2000; 4: CD000364.

25.- POOLE P J, CHACKO E, WOOD-BAKER R W, CATES C J. Influenza vaccine for patients with chronic obstructive pulmonary disease. Cochrane Database Syst Rev 2000; 4: CD002733.

26.- NICHOL K L, BAKEN L, WUORENMA J, NELSON A. The health and economic benefits associated with pneumococcal vaccination of elderly persons with chronic lung disease. Arch Intern Med 1999; 159: 2437-42.

27.- CHRISTENSON B, LUNDBERGH P, HEDLUND J, ÖRTQVIST A. Effects of a large-scale intervention with influenza and 23-valent pneumococcal vaccines in adults aged 65 years or older: a prospective study. Lancet 2001; 357: 1008-11.

28.- CHRISTENSON B, HEDLUND J, LUNDBERGH P, ÖRTQVIST A. Additive preventive effect of influenza and pneumococcal vaccines in elderly persons. Eur Respir J 2004; 23: 363-8. 